\title{
Sports Biomechanics
}

\section{Performance of disabled swimmers in protocols or tests and competitions: a systematic review and meta-analysis}

\section{Wellington G. Feitosa, Ricardo de Assis Correia, Tiago M. Barbosa \& Flávio A.}

de Souza Castro

To cite this article: Wellington G. Feitosa, Ricardo de Assis Correia, Tiago M. Barbosa \& Flávio A. de Souza Castro (2019): Performance of disabled swimmers in protocols or tests and competitions: a systematic review and meta-analysis, Sports Biomechanics, DOI: 10.1080/14763141.2019.1654535

To link to this article: https://doi.org/10.1080/14763141.2019.1654535

曲 Published online: 27 Sep 2019.

Submit your article to this journal $₫$

Q View related articles $₫$

View Crossmark data ¿ 


\title{
Performance of disabled swimmers in protocols or tests and competitions: a systematic review and meta-analysis
}

\author{
Wellington G. Feitosa (D) ${ }^{a, b}$, Ricardo de Assis Correia (D) ${ }^{a}$, Tiago M. Barbosa (D) ${ }^{c, d, e}$ \\ and Flávio A. de Souza Castro (iD ${ }^{a}$
}

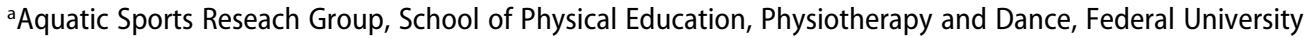

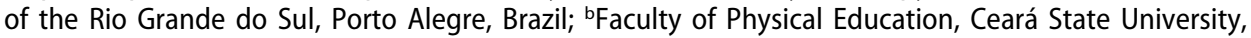
Fortaleza, Brazil; cPhysical Education and Sport Science Academic Group, National Institute of Education, Nanyang Technological University, Singapore, Singapore; dDepartment of Sport Sciences, School of

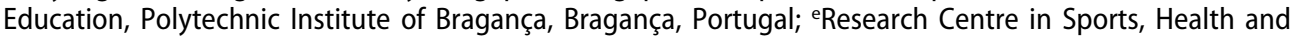
Human Development, Vila Real, Portugal

\begin{abstract}
Biomechanical and coordination measurements are useful tools to assess swimming performance. Regarding Paralympic swimming, function and technique make these measurements complex. The aim of this study was to perform a systematic review of studies on biomechanics, coordination and performance in disabled swimmers following swimming protocols and in competitions. Data sources: PubMed, EMBASE, ISI Web of Knowledge, SPORTDiscus and Academic Search Premier. We selected complete studies, published until June 2018. Eighteen studies satisfied the inclusion criteria and were selected for qualitative analysis; nine of these were included in the meta-analysis. Swimming speed and stroke length increase with less impact of physical, visual and intellectual impairment on performing specific swimming tasks. Stroke rate is more stable all through the sport classes than both swimming speed and stroke length. Most physically disabled swimmers adopt the catch-up coordination model. Stroke rate is responsible for most of the intracycle velocity variation in swimmers with amputations or malformations of the upper limbs. No study was found on propulsive efficiency. Swimmers with disabilities should work more on stroke rate, with small decreases in stroke length to achieve higher swimming speeds, lower swim coordination index (more negative) and lower speed variations.
\end{abstract}

\section{ARTICLE HISTORY}

Received 6 November 2018

Accepted 5 August 2019

\section{KEYWORDS}

Swimming; classification; paralympics; disability sport; front crawl

\section{Introduction}

Due to the particularities of swimming, mastering the technique plays a decisive role in performance (Almena, Pérez-Tejero, Coterón, \& Veiga, 2015). These particularities influence the performance of swimmers with disabilities (SWD), for example, regarding biomechanical aspects during swimming protocols or tests, for the physically (Oh, Burkett, Osborough, Formosa, \& Payton, 2013; Osborough, Daly, \& Payton, 2015), visually (Malone, Sanders, Schiltz, \& Steadward, 2001; Souto, Oliveira, \& Santos Filho, 2016) or intellectually disabled. Although studies with Paralympic swimmers have increased in 
number, possibly due to the visibility of Paralympic sports (Sousa, Corredeira, \& Pereira, 2013), analyses that bring together a body of evidence can help to better understand the performance parameters in Paralympic swimming.

There are different biomechanical parameters for swimming analysis. Among them, kinematic parameters, such as (i) clean swimming speed, (ii) stroke length and (iii) stroke rate, can provide useful information about technique and for the classification system in competitive swimming in a less complex way (Daly, Djobova, Malone, Vanlandewijck, \& Steadward, 2003). Parameters that require more accurate techniques are (i) intra-cyclic swimming velocity variation (Barbosa et al., 2005, 2013); (ii) coordination parameters, such as the latency time of the upper limbs' propulsion actions, represented by the index of coordination for swimmers without disabilities in the upper limbs (Chollet, Chalies, \& Chatard, 2000; Figueiredo, Seifert, Vilas-Boas, \& Fernandes, 2012); and (iii) the adapted index of coordination for those with amputation or malformation in only one upper limb (Osborough, Payton, \& Daly, 2010; Willig et al., 2012). Moreover, kinetics parameters, such as propulsive efficiency (Zamparo, Pendergast, Termin, \& Minetti, 2002), and the force measured during tethered swimming (Morouço, Keskinen, Vilas-Boas, \& Fernandes, 2011) can provide important information in the context of competitive swimming.

Swimmers with disabilities are grouped into categories by impact of an eligible impairment on their ability to perform the specific and fundamental tasks of the sport (except for swimmers with visual impairment according to the (International Paralympic Committee [IPC], 2017): minimum impairment criteria must take into consideration the use of adaptive equipment). Some assessment sessions are conducted to classify the swimmers, e.g.: (i) functional assessment (assesses whether a swimmer complies with minimum impairment criteria to compete in one of the 10 sport classes for SWD); (ii) medical assessment (three sport classes for swimmers with vision impairment (iii) technical assessment (assesses the extent to which an athlete is able to execute the specific tasks of competitive swimming); and (iv) observation in competition assessment.

Additionally, swimmers with an intellectual impairment (sport class S14) have a restriction in intellectual functioning and adaptive behaviour that affects the conceptual, social and practical adaptive skills needed for everyday life. Swimmers must meet the eligibility criteria of the International Federation for Athletes with Intellectual Impairments (INAS) and complete the Training History and Sport Limitation Questionnaire (TSAL-Q) provided by World Para Swimming. Thus, swimmers with intellectual impairments must pass through the sport cognition test battery and, if required, observation in competition assessment (IPC, 2017).

Classification in Paralympic sports should be based on function and not performance (Tweedy \& Vanlandewijck, 2011). However, it is common to question whether these swimmers' Paralympic classification is fair or not based on performance characteristics (Burkett et al., 2018; Daly, Malone, Vanlandewijck, \& Steadward, 1999a; Malone et al., 2001; Oh et al., 2013). In this sense, it is possible to develop analyses based on the relation between swimming performance biomechanical parameters and the Paralympic categories according to their results in protocols and official swimming competitions available in scientific databases. In this sense, assessing performance parameters (biomechanical, coordination and performance) can add information to the context of adapted sport. 
Furthermore, there is a large diversity of disabilities and number of sport classes, a small number of participants with similar disabilities, and different protocols used to assess the biomechanical performance of these swimmers. A preliminary search of the main databases (PubMed, EMBASE, ISI Web of Knowledge, SPORTDiscus and Academic Search Premier) provided 478 studies on the participants. Information on the biomechanical parameters for SWD found in these studies is pertinent to the development of scientific knowledge in the adaptive sports field. In addition, it is of great value for Paralympic swimming to continue evolving in a specialised way in the sport scene, in training and in competitions. Thus, gathering a set of studies that demonstrate evidence on performance parameters in swimming is a great challenge, but it can contribute to Paralympic sports, the scientific community, teachers, coaches, technicians and, especially, swimmers. Thus, the aim of this study was to perform a systematic review of studies on biomechanics, coordination and performance in disabled swimmers following swimming protocols and competitions.

\section{Methods}

This systematic review and meta-analysis was developed according to the recommendations of the PRISMA Statement (Vrabel, 2015).

\section{Eligibility criteria}

This review included studies that recruited swimmers with physical, motor, visual or intellectual disabilities who were exposed to any biomechanical assessment (swimming speed, stroke length and rate, speed variation, index of coordination and adapted index of coordination, propelling efficiency and tethered force). Observational studies with swimming protocols, tests and competitions were included (with any follow-up and published in the full version). There was no language restriction, as long as the studies had titles and abstracts in English. Exclusion criteria were: lack of data and/or incomplete data, and multiple publications with repeated results (only one study included).

\section{Research sources}

The studies contained in this systematic review were found in the following electronic databases: PubMed, EMBASE, ISI Web of Knowledge, SPORTDiscus and Academic Search Premier. In addition to the searches in the databases, the references of the articles found were also searched, to identify additional studies that were not found with the previous strategy, e.g. complete articles, theses and dissertations containing relevant and original information.

\section{Research strategy}

The research strategy used (i) the Medical Subject Headings (MeSH) terms and (ii) the EMBASE platform EMTREE terms: 'Sports for Persons with Disabilities', 'Adaptive Sport', 'Para-Athletic', 'Disabled Persons, 'Person, Disabled', 'Disabled Sport', 'Persons with Disability', 'Handicapped', 'Little Persons', 'Physically Disabled', 'Disabled, 
Physically', 'Physically Challenged', 'Amputee', 'Visually Impaired Persons', 'Impaired Person, Visually', 'Impaired Persons, Visually', 'Person, Visually Impaired', 'Visual Impairment', 'Blind Person', 'Person, Blind', 'Intellectual Disability', 'Disabilities, Intellectual', 'Intellectual Disabilities', 'Mental Retardation', 'Disability, Intellectual', 'Intellectual Development Disorder', 'Cerebral Palsy' and 'Swimming' and their synonyms and combinations, in complete longitudinal studies published by 1 June 2018. The Boolean operators 'AND' and 'OR' were used for tracing during the searches in the electronic databases.

\section{Results extraction}

The search was carried out by two people together. The other phases were performed independently. In the first phase, two researchers, in duplicate, independently assessed the titles and abstracts of all the articles identified. All abstracts that did not provide sufficient information regarding the inclusion and exclusion criteria were selected for assessment of the full paper. In the second phase, the same reviewers independently assessed the entire articles and made their selection according to the eligibility criteria. A third independent reviewer resolved disagreements between reviewers. The two reviewers extracted the data with a standard form.

\section{Biomechanical outcomes}

The selected biomechanical outcomes were: (i) clean swimming speed (SS), which does not consider start, turn and finish times (Daly et al., 1999a); (ii) stroke rate (SR), the number of stroke cycles per time unit; (iii) stroke length (SL), the average distance travelled by the body in each stroke cycle (Craig \& Pendergast, 1979; Hay \& Guimarães, 1983); (iv) intra-cyclic velocity variation (IVV), the kinematic consequence of the nonhomogeneous distribution of propulsion over time (Barbosa et al., 2006); (v) the index of coordination (IdC) and adapted index of coordination (IdCadapt), respectively, for swimmers non-disabled and disabled in upper arm, that is, the interval between the propulsive phases of the strokes (Chollet et al., 2000). The IdCadapt occurs when there is a single upper-limb amputation, and from a common point on both arms for swimmers without disabilities in the upper limbs (Osborough et al., 2010).

The IdC and IdCadapt have three models: capture ( $\mathrm{IdC}<0 \%)$, characterised by a gliding phase; opposition ( $\mathrm{IdC}=0 \%$ ), in which the propulsion of one arm begins exactly when the propulsion of the other arm ends; and overlap ( $\mathrm{IdC}>0 \%$ ), when the propulsive phases of the two arms overlap. Also measured were: (vi) propelling efficiency (PE) which expresses the mechanical power required to overcome drag in relation to the total mechanic power generated (Martin, Yeater, \& White, 1981; Zamparo, 2006); and (vii) the force measured during tethered swimming, assumed to be representative of the propulsive forces applied by the swimmer (Keskinen \& Komi, 1993).

\section{Assessing risk of bias}

Two reviewers independently assessed the methodological quality of the articles. The Downs and Black Quality Assessment Checklist allows assessment of the quality of 
randomised and non-randomised studies (cohort and case-control studies) from an overall score (Downs \& Black, 1998). The main items of quality assessment are reports, internal and external validity (bias and confusion) and power. The original version has 27 items. However, some adaptations were made to better fit the focus of this research: (i) item 27 was not used for evaluating whether the negative findings from the study could be due to chance; (ii) replacement of the words 'patient' by 'participant' and 'treatment' by 'testing'. Previous studies have made similar adaptations (Costa, Balasekaran, Vilas-Boas, \& Barbosa, 2015; Hebert-Losier, Supej, \& Holmberg, 2014; Mills, Hunt, \& Ferber, 2013). Articles with higher scores are considered to be of superior quality. These scores were converted into percentage values: [(total number of points reached/total number of applicable points $) \times 100]$.

\section{Data analysis}

Quantitative analysis was performed based on the main characteristics of the studies: authors' names and year of publication, data sampling, and main methods with selected outcomes. The meta-analysis was performed using a random effect model (Borenstein, Hedges, Higgins, \& Rothstein, 2010). The inconsistency test $\left(\mathrm{I}^{2}\right)$ assessed the statistical heterogeneity among the studies, in which the values represent heterogeneities: null $=$ close to $0 \%$; low $=$ close to $25 \%$; moderate $=$ close to $50 \%$; high $=$ close to $75 \%$ (Borenstein, Hedges, Higgins, \& Rothstein, 2009). Alpha was set at 0.05. The risk of bias analyses were performed through a forest plot in OpenMeta[Analyst] software (Wallace, Schmid, Lau, \& Trikalinos, 2009). Sensitivity analyses were performed by subgroup, with the addition of the covariant 'Sport Class', according to the characteristics of the studies (Viechtbauer, 2010).

\section{Results}

\section{General}

The search strategy identified 478 articles; 280 were duplicates; seven studies were included from other sources (found in the references of articles, specialised books and articles sent by courtesy of the author). In the first phase, 176 studies were excluded after analysis of the titles and abstracts. In the second phase, 12 studies were excluded after full reading. In total, 18 articles were examined extensively because they fitted the previously established inclusion criteria; protocols or tests were found for several distances (Tables 1, 2 and 3). However, no study was found with competitive events longer than $100 \mathrm{~m}$. Finally, only nine studies (100 m competitive events) were included in the meta-analysis. These studies assessed the selected parameters at (Figure 1):

(i) Atlanta 1996 Paralympic Games (Daly et al., 1999a; Malone et al., 2001);

(ii) During the 1995 European Championships (Pelayo, Sidney, Moretto, Wille, \& Chollet, 1999);

(iii) The Global Games in 2004 with data taken from Sydney 2000 Paralympic Games (Daly et al., 2003; Daly, Einarsson, Van de Vliet, \& Vanlandewijck, 2006; Daly, Malone, Burkett, Gabrys, \& Satkunskiene, 2009); 


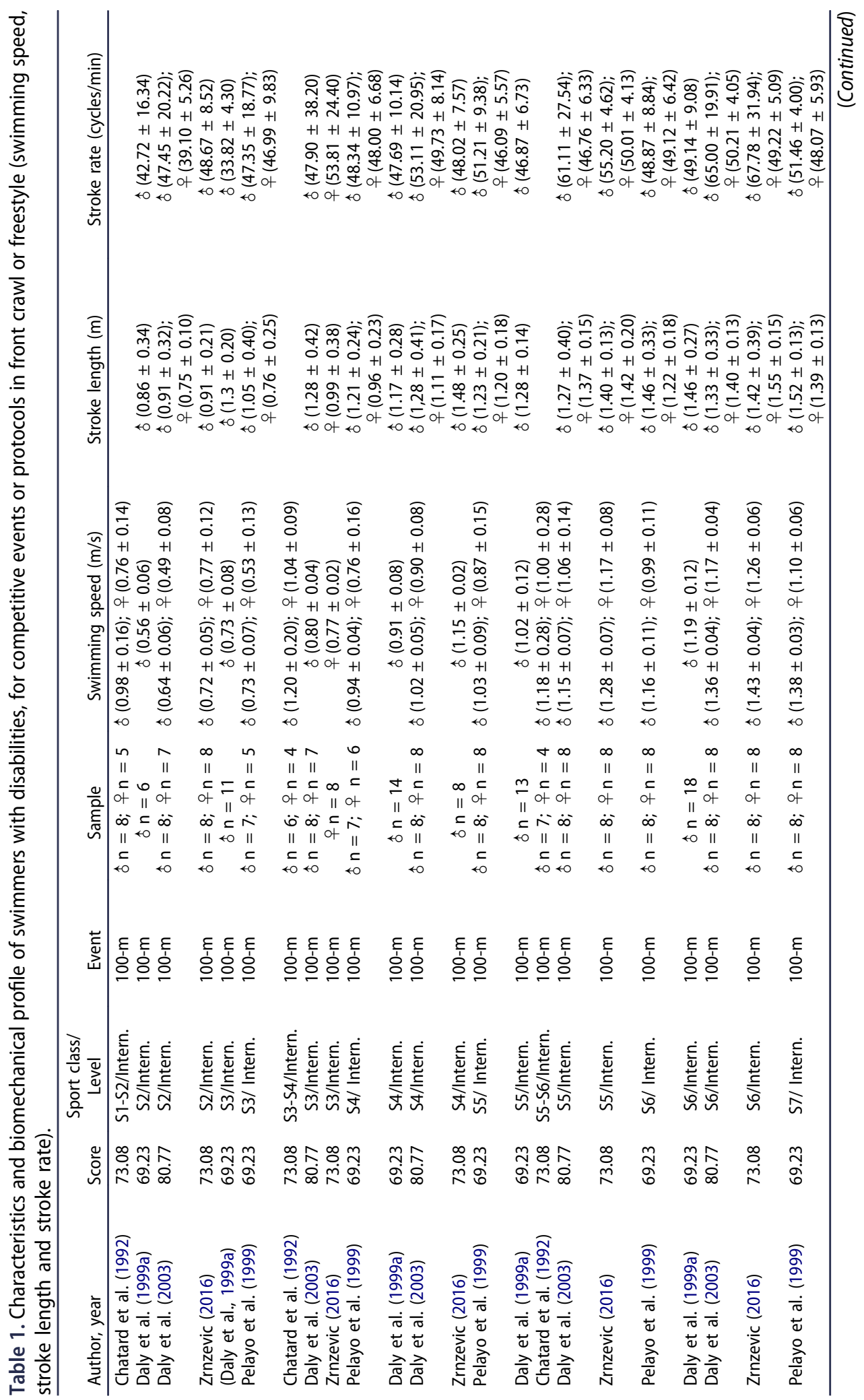




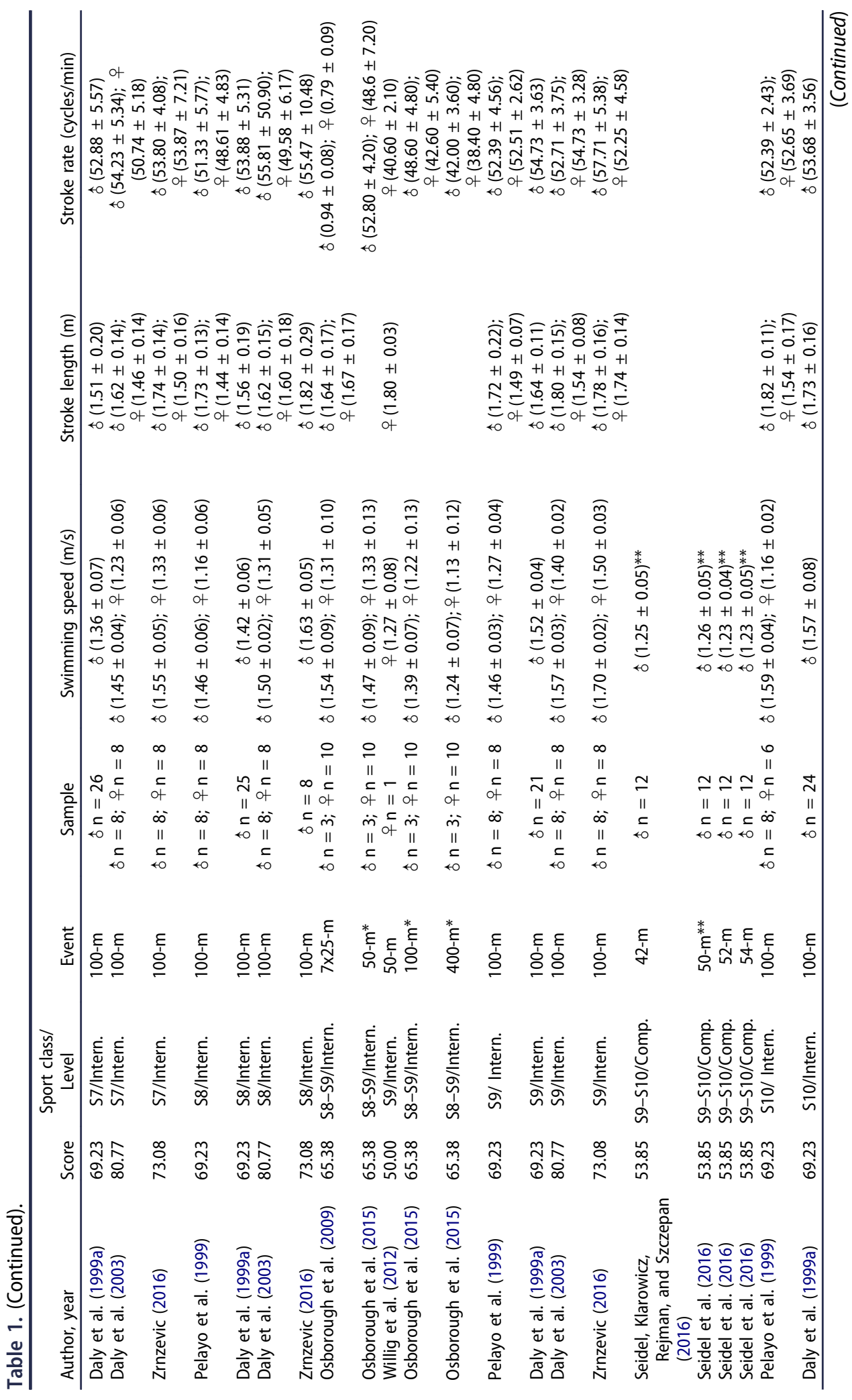




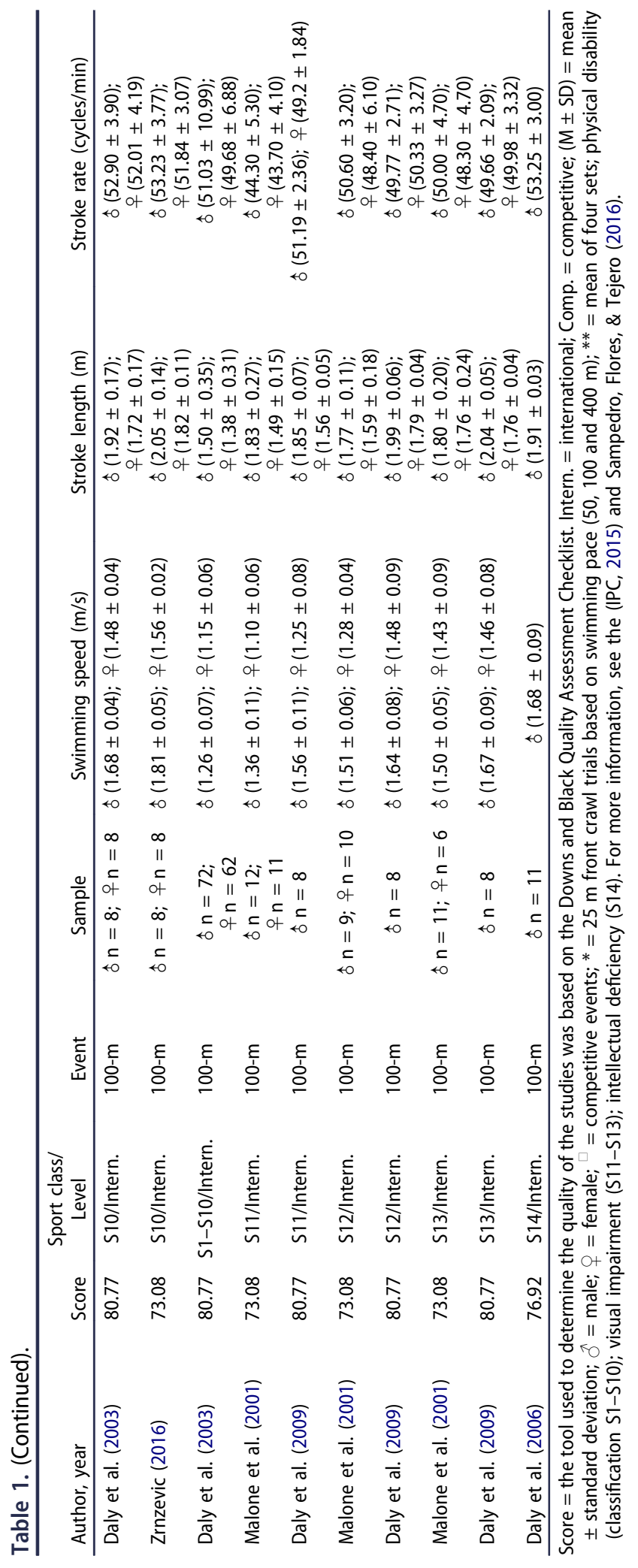




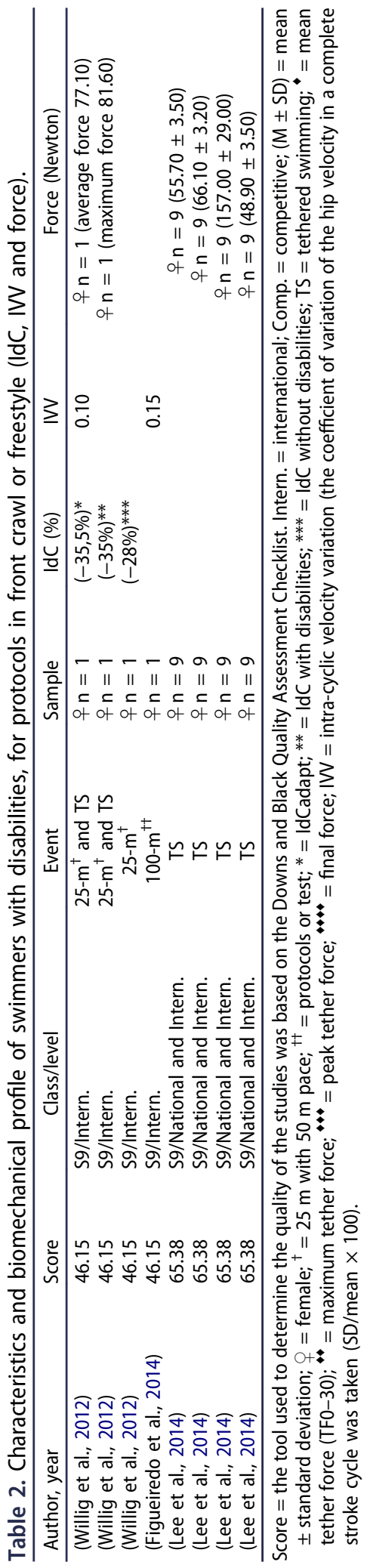




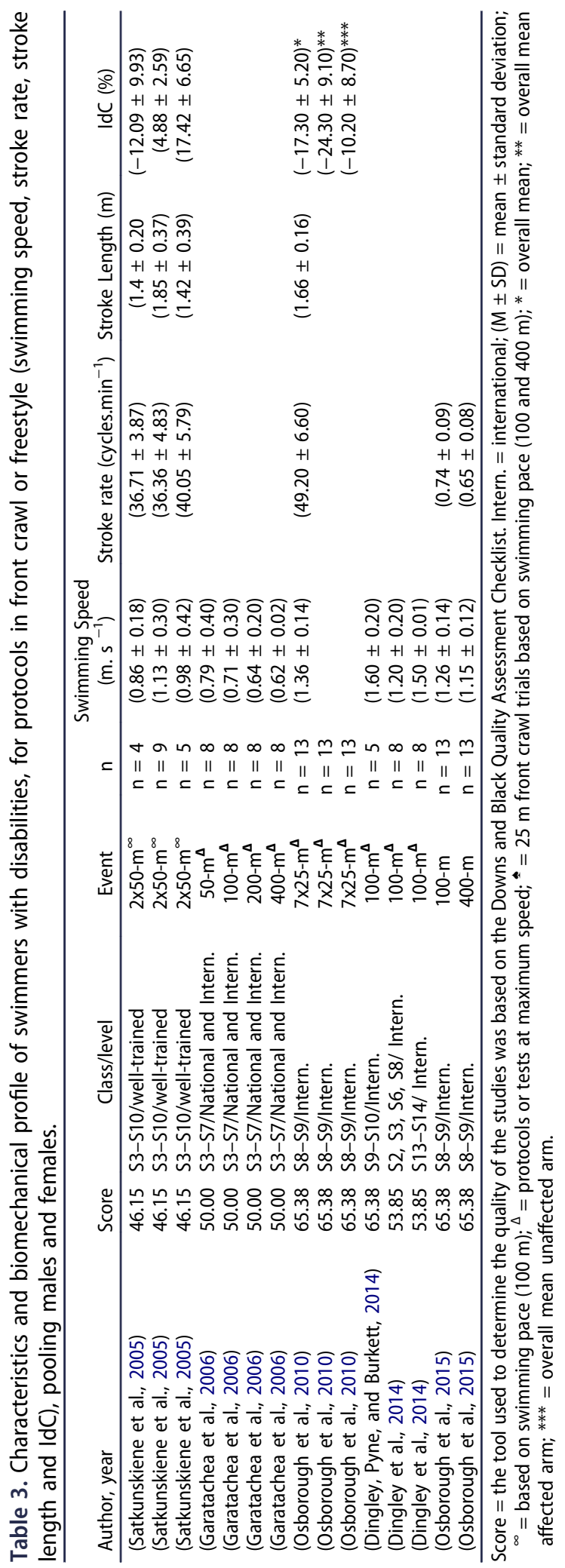




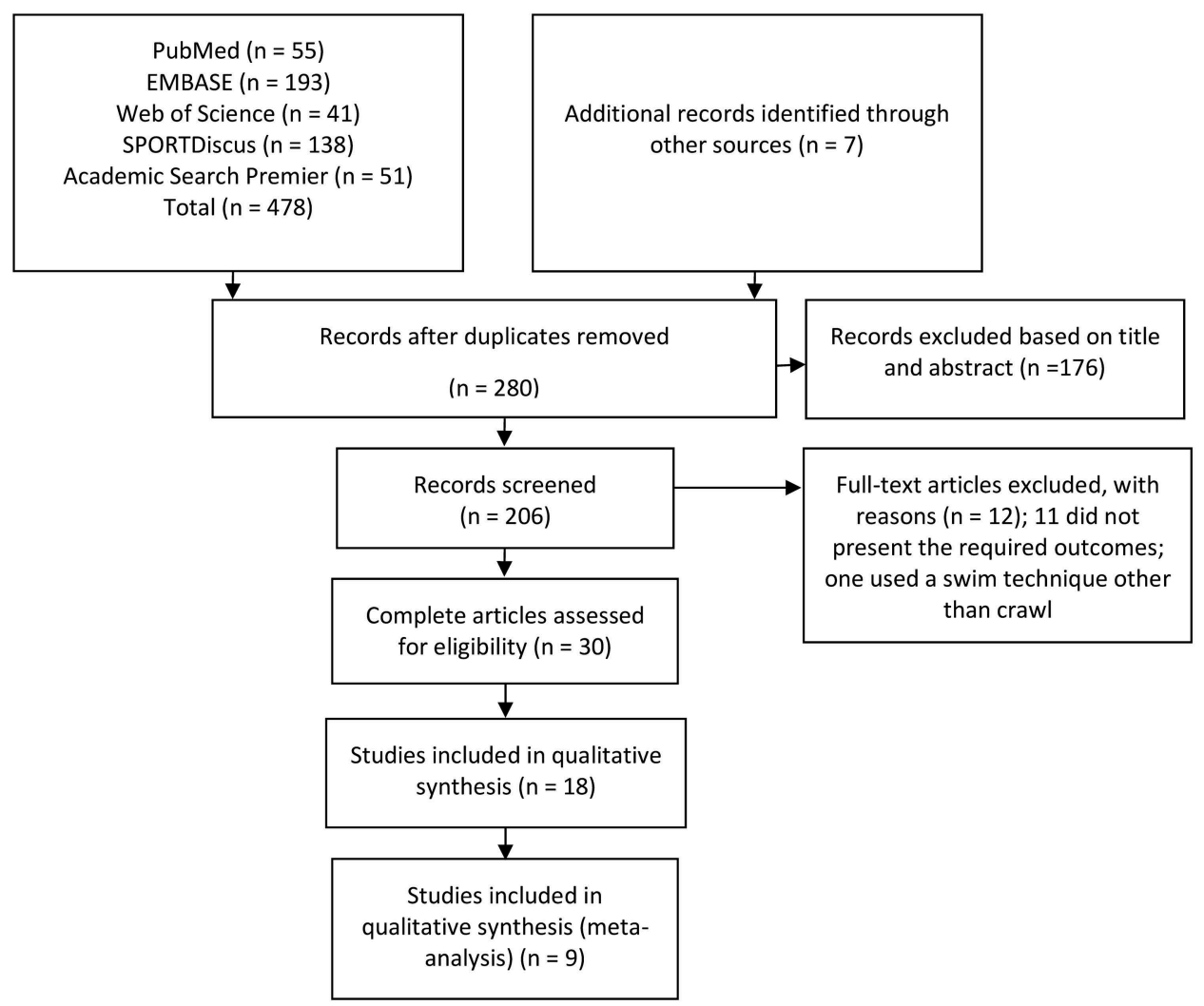

Figure 1. Flowchart of the systematic literature search.

(iv) London 2012 Paralympic Games (Zrnzevic, 2016);

(v) $27^{\text {th }}$ International German Championships for Swimmers with an Impairment (race kinematical analysis at IDM BERLIN 2013) (Willig et al., 2012); this study was published in an issue (year) that was delayed due to internal reasons of the journal, according to information from the authors of the article and the publication's website;

(vi) One study does not make any reference to competitive events for internationallevel swimmers (Chatard et al., 1992).

The authors' names, year of publication, quality score of the studies, whether assessed in protocols or tests and competitions, and elective outcomes for the present study are shown in Table 1 . The percentage quality of the articles included ranged from $50.00 \%$ to $80.77 \%$, with an average of $64.32 \pm 11.56 \%$ (according to the Downs and Black Quality Assessment Checklist) (Tables 1, 2 and 3).

The biomechanical parameters found and number of studies are: i) SS $=16$ (male $\mathrm{n}=501$ and female $\mathrm{n}=306$ ), $\mathrm{SR}=12$ (male $\mathrm{n}=465$ and female $\mathrm{n}=283$ ), $\mathrm{SL}=11$ (male $\mathrm{n}=462$ and female $\mathrm{n}=258$ ). The biomechanical parameters SS, SR and SL were found more widely in the eligible articles in the $100 \mathrm{~m}$ freestyle race, which enabled statistical analysis (meta-analysis); ii) IdC and IdCadapt = three studies with 
different characteristics $(\mathrm{n}=32)$ that make it difficult to compare them: (Satkunskiene, Schega, Kunze, Birzinyte, \& Daly, 2005), pooling males and females (sport classes S3 to S10, $\mathrm{n}=18$; group $1(\mathrm{n}=4)=$ sport classes $2 \times \mathrm{S} 4,2 \times \mathrm{S} 9$; group $2(n=9)=$ sport classes S5, S6, $2 \times$ S7, S8, S9 and $3 \times$ S10; group $3(n=5)=$ sport classes S3, S4, S6 and S7) in $2 \times 50 \mathrm{~m}$ freestyle; Osborough et al. (2010), pooling males and females $(n=13$, sport classes S8 and S9) in $7 \times 25$ m tests with 3 min intervals (group 1: slow speed to maximum speed; group 2: maximum speed to slow speed); and Willig et al. (2012), with only one swimmer (sport class S9) in $25 \mathrm{~m}$ freestyle with pace of $50 \mathrm{~m}$; iii) IVV = two studies with the same female swimmer $(\mathrm{n}=1)$ in different tests, IVV $=0.10$ for $25 \mathrm{~m}$ with pace of $50 \mathrm{~m}$ (Willig et al., 2012) and IVV $=0.15$ in $100 \mathrm{~m}$ (Figueiredo, Willig, Alves, Vilas-Boas, \& Fernandes, 2014); iv) tethered swimming force = only two studies were found, with female swimmers ( $\mathrm{n}=10$, sport class S9) in a $30 \mathrm{~s}$ test on tethered swim force kinetics. The study by Willig et al. (2012; $\mathrm{n}=1$ ), presented higher results for strength (average force $77.10 \mathrm{~N}$; maximum $81.60 \mathrm{~N}$ ) when compared to the study by Lee et al. (2014; $\mathrm{n}=9$; average force $55.70 \pm 3.50 \mathrm{~N}$; maximum force $66.10 \pm 3.20 \mathrm{~N})$. However, the first study (Willig et al., 2012) deals with a single case, which makes statistical comparisons unfeasible.

The studies were separated into biomechanical outcomes of swimmers by gender (Tables 1 and 2) and biomechanical outcomes of grouped male and female disabled swimmers (Table 3). No study was found with the PE outcome.

\section{Meta-analysis of the observational studies}

\section{Swimming speed}

The SS meta-analysis showed higher values the higher the sport class, in the $100 \mathrm{~m}$ front crawl trials for male $(\mathrm{n}=444)$ and female $(\mathrm{n}=240)$ SWD, sport classes S1-S14 for males, and sport classes S1-S13 for females (Figure 2) for physical disability (sport classes S1-S10), visual impairment (sport classes S11-S13) and intellectual disability (sport class S14). For more information, see (IPC, 2015; Sampedro, Flores, \& Tejero, 2016). The estimated mean SS for men and women, respectively, were 1.29 and $1.13 \mathrm{~m} / \mathrm{s}$ (95\% CI: $1.21-1.38$ and 1.03-1.23, respectively; standard error: 0.04 and $0.05, \mathrm{p}<0.01 ; \mathrm{I}^{2}>75 \%, \mathrm{p}<0.01$ ), when all studies and respective sport classes were considered together.

For SS, the sensitivity analysis in the male and female subgroups indicated high heterogeneity ( $\mathrm{I}^{2}$ close to $75 \%, \mathrm{p}<0.05$ ) for comparisons S1-S2, S2-S2, S3-S3, S4-S4, S5-S5, S6-S6, S7-S7, S8-S8, S9-S9 and S10-S10 and between sport classes, e.g. S3-S4, S4-S5, S5-S6, S6-S7, S7-S8, S8-S9, S9-S10, S11-S13, S11-S12 and S12-S13, within the $100 \mathrm{~m}$. For swimmers with an intellectual disability there was no comparison by subgroup (there was only one sport class).

\section{Stroke length}

SL was also higher in the sport classes with a lower degree of disability in $100 \mathrm{~m}$ front crawl in male $(\mathrm{n}=423)$ and female $(\mathrm{n}=219)$ SWD (Figure 3). When considering all studies and their respective sport classes together, the estimated mean SL for men was 1.56 m (95\% CI: 1.48-1.63; standard error: 0.04, $\left.\mathrm{p}<0.01 ; \mathrm{I}^{2}>75 \%, \mathrm{p}<0.01\right)$, while for 


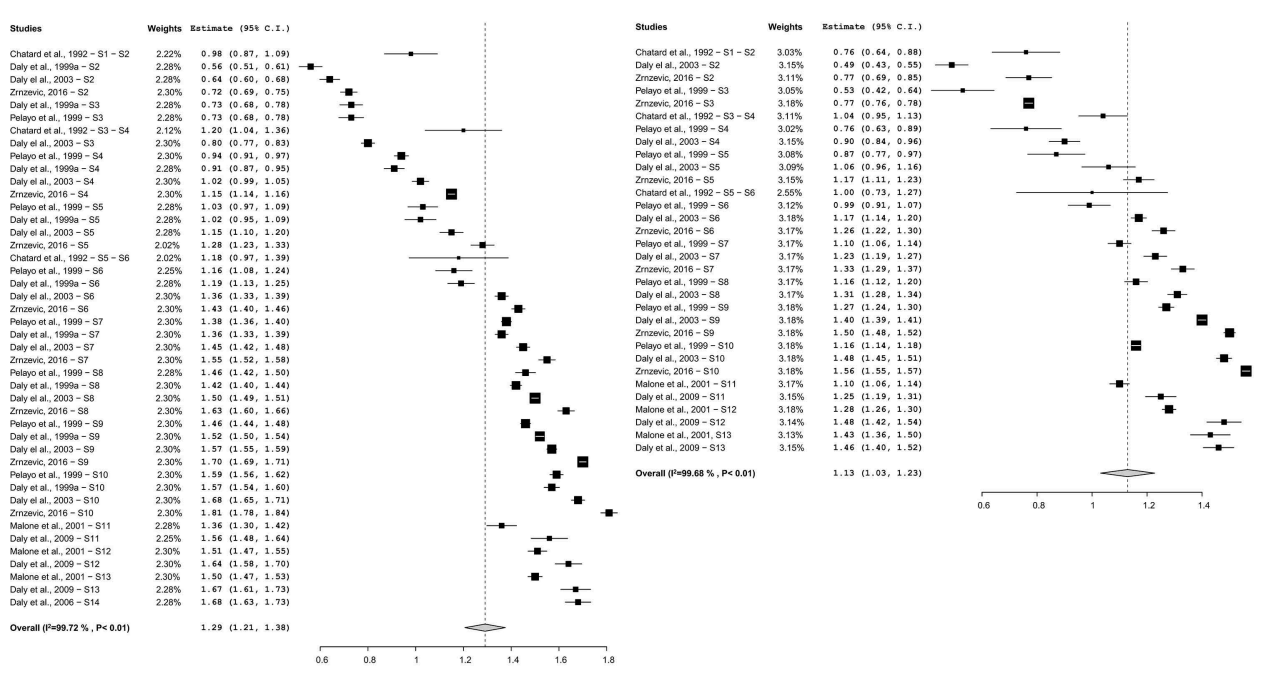

Figure 2. Forest plots derived from continuous random-effects models depicting the swim speed by Paralympic classification (sport classes S1 to S14 for male and S1-S13 for female) in $100 \mathrm{~m}$ freestyle. Right and left, respectively: male forest plot and female forest plot.
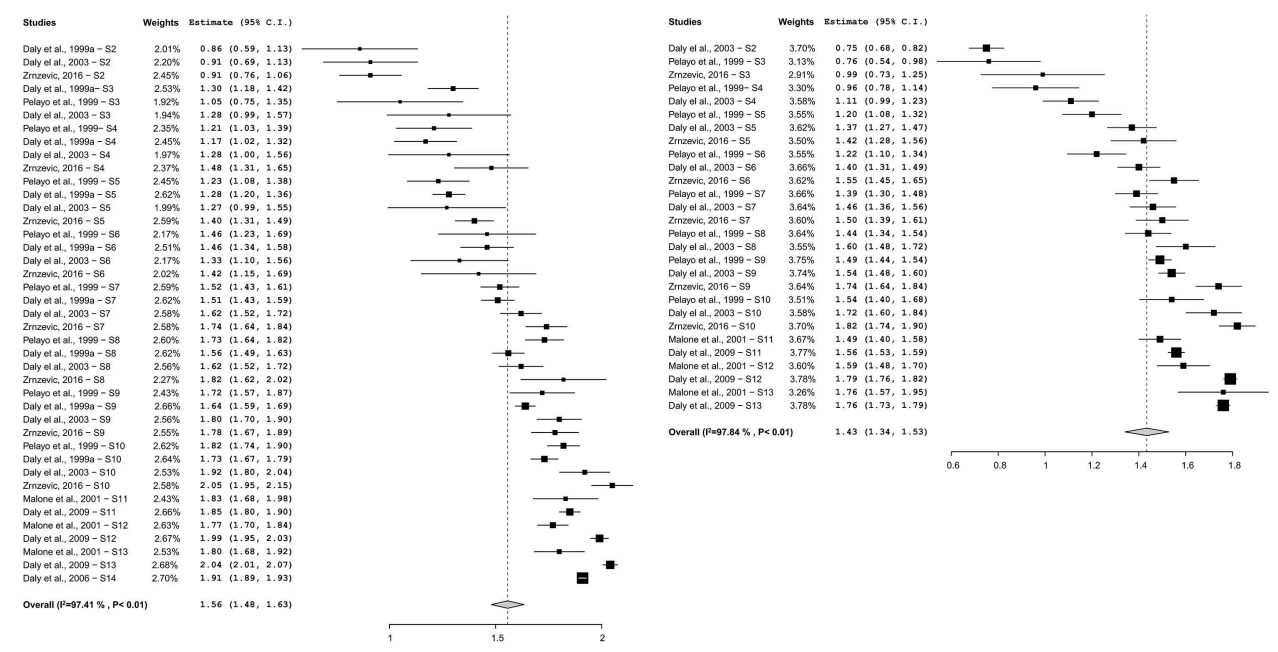

Figure 3. Forest plot derived from continuous random-effects models depicting stroke length $(\mathrm{m})$ by Paralympic classification (sport classes S2 to S14, male) in competitions of $100 \mathrm{~m}$ freestyle. Right and left, respectively: male forest plot and female forest plot.

women, the estimated mean SL was $1.43 \mathrm{~m}$ (95\% CI: 1.34-1.53; standard error: 0.05, $\left.\mathrm{p}<0.01 ; \mathrm{I}^{2}>75 \%, \mathrm{p}<0.01\right)$.

The SL sensitivity analysis for the male subgroup showed no heterogeneity $\left(\mathrm{I}^{2}=0 \%\right.$, $\mathrm{p}>0.05$ ) for sport classes S2 and S6 and for the comparisons within sport class S11, in the $100 \mathrm{~m}$; low heterogeneity ( $\mathrm{I}^{2}$ close to $25 \%, \mathrm{p}>0.05$ ) for studies with sport class $\mathrm{S} 3$, in the $100 \mathrm{~m}$; moderate heterogeneity $\left(\mathrm{I}^{2}\right.$ close to $\left.50 \%, \mathrm{p}<0.05\right)$ for the comparisons $\mathrm{S} 4$, $\mathrm{S} 5$ and $\mathrm{S} 3-\mathrm{S} 6$, in the $100 \mathrm{~m}$; high heterogeneity $\left(\mathrm{I}^{2}\right.$ close to $\left.75 \%, \mathrm{p}<0.05\right)$ for the 
comparisons of sport classes S7, S8, S9, S10, S5-S6, S6-S7, S7-S8, S8-S9, S9-S10, S11$\mathrm{S} 12$ and S12-S13, in the $100 \mathrm{~m}$. The SL sensitivity analysis for the female subgroup indicated no heterogeneity $\left(\mathrm{I}^{2}=0 \%, \mathrm{p}>0.05\right)$ for studies with sport class $\mathrm{S} 13$, within the $100 \mathrm{~m}$; and low heterogeneity ( $\mathrm{I}^{2}$ close to $25 \%, \mathrm{p}>0.05$ ) for studies with sport classes S2-S3 and S7, in the $100 \mathrm{~m}$. Moderate heterogeneity $\left(\mathrm{I}^{2}\right.$ close to 50\%, $\left.\mathrm{p}<0.05\right)$ was found for sport classes $\mathrm{S} 4$ and $\mathrm{S} 11$, in the $100 \mathrm{~m}$. High heterogeneity ( $\mathrm{I}^{2}$ close to $75 \%, \mathrm{p}<0.05)$ was found for sport classes S5, S6, S8, S9 and S10, in the $100 \mathrm{~m}$.

\section{Stroke rate}

The SR was higher in male swimmers in sport classes with lower disability levels, in $100 \mathrm{~m}$ front crawl for male swimmers $(\mathrm{n}=423)$ and for most female swimmers $(\mathrm{n}=219)$ (Figure 4). The estimated mean SR for men and women were, respectively, 51.10 and 49.55 cycles/min (95\% CI: $49.71-52.50$ and 48.38-50.72; standard error: 0.71 and $\left.0.60, \mathrm{p}<0.01 ; \mathrm{I}^{2}>75 \%, \mathrm{p}<0.01\right)$ when all studies and respective sport classes were considered together.

The SR sensitivity analysis for the male subgroup showed no heterogeneity $\left(\mathrm{I}^{2}=0 \%\right.$, $\mathrm{p}>0.05)$ for the studies with sport classes S7-S10 and S12-S13, in the $100 \mathrm{~m}$; and low heterogeneity ( $\mathrm{I}^{2}$ close to 25\%, $\mathrm{p}>0.05$ ) for studies with S2-S6 except for (Daly et al., 1999a), in the $100 \mathrm{~m}$. The study by Daly et al. (1999a) found that sport class S3 for SR increases the heterogeneity of any study, because it has a very low SR in relation to other studies, so it was removed from the calculation of sport classes S2-S6, in the $100 \mathrm{~m}$. High heterogeneity $\left(\mathrm{I}^{2}\right.$ close to $75 \%, \mathrm{p}<0.05$ ) was found for sport classes S11-S13, in the $100 \mathrm{~m}$. The SR sensitivity analysis for the female subgroup showed no heterogeneity $\left(\mathrm{I}^{2}=0 \%, \mathrm{p}>0.05\right)$ for the studies with sport classes S3-S8 and S9-S10, in the $100 \mathrm{~m}$; and with sport classes S12-S13 in the $100 \mathrm{~m}$. Moderate heterogeneity ( $\mathrm{I}^{2}$ close to $50 \%, \mathrm{p}>0.05$ ) was found for sport classes $\mathrm{S} 8-\mathrm{S} 10$, in the $100 \mathrm{~m}$; high heterogeneity $\left(\mathrm{I}^{2}\right.$ close to $\left.75 \%, \mathrm{p}<0.05\right)$ was found for sport classes $\mathrm{S} 11-$ $\mathrm{S} 12$, in the $100 \mathrm{~m}$, and for sport classes $\mathrm{S} 11-\mathrm{S} 13$, in the $100 \mathrm{~m}$.
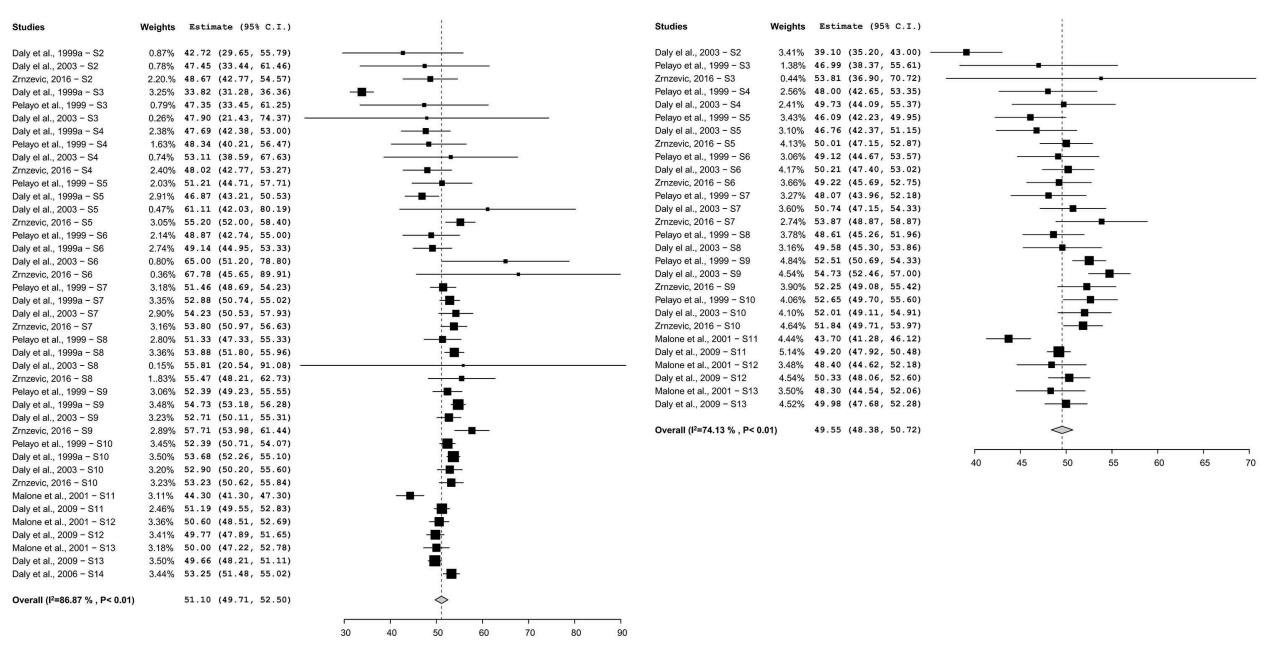

Figure 4. Forest plot derived from continuous random-effects models depicting the stroke rate (cycle/min) by Paralympic classification (sport classes S2 to S14, male) in competitions of $100 \mathrm{~m}$ freestyle. Right and left, respectively: male forest plot and female forest plot. 


\section{Discussion and implications}

This research carried out a systematic review and meta-analysis of studies that examined kinematics, coordination and kinetics in swimmers with physical, visual and intellectual disabilities, in protocols, tests and swimming competitions. The main findings of the meta-analysis are twofold: (i) SS, SL and SR are higher the lower the swimmers' disability level; (ii) comparisons of classifications did not present statistical heterogeneities and differences for: a) SL in sport classes S2, S6 and S11 in the $100 \mathrm{~m}$ for males, and sport class S13 in the $100 \mathrm{~m}$ for females; b) SR in sport classes S7-S10 and $\mathrm{S} 12-\mathrm{S} 13$ in the $100 \mathrm{~m}$ for males, and sport classes S3-S8, S9-S10 and S12-S13 in the $100 \mathrm{~m}$ for females.

Studies with better quality assessments fill in all the fields in the quality checklist (Downs \& Black, 1998), except for the domain internal validity-confounding (selection bias) which was not filled by any study. The internal validity-confounding (selection bias) domain deals with bias in the selection of study participants (all non-randomised studies should receive a zero percentage). On the other hand, studies with smaller percentages achieved in the assessment of quality in details, show as minor items, e.g., Reporting, items 3 and 8, concerning clarity in the description of the characteristics of participants and reporting adverse events that may be a result of the intervention; External validity, item 11representativeness of the participants in the population from which they were recruited (studies with a single case, $\mathrm{n}=1$, obtained lower scores); Internal validity-bias, items 14 and 15-regarding blinding of the participants and those measuring the main outcomes of the intervention. It is necessary to emphasise that for the results on video collections of competitions, the blinding of participants is probably irrelevant (there were positive scores in these cases). Regarding the people who measure the outcomes by video collection, it is difficult to indicate whether or not there was blinding (in these cases, the score was zero).

In this way, the articles assessed in this study had lower quality than those in other scientific fields, e.g., health science, biomechanics and performance (Ferreira, Barbosa, Costa, Neiva, \& Marinho, 2016; Hebert-Losier et al., 2014; Mills et al., 2013). These factors could be better developed in future investigations (filling in the missing issues and developing instruments appropriate to that population), but the difficulty in conducting research with SWD is also evident.

The results found in the meta-analysis for SS, SL and SR seem to be in agreement with the Paralympic functional classification for swimmers in the same sport class and with similar swimming performance. Thus, the SR parameter is more unstable among sports classes, although there is a slight increase from lower to higher sport classes. In the case of physically impaired swimmers, there are 10 sport classes, which shows great morphological variation (Oh et al., 2013). The SS values were different among all sport classes for swimmers with physical disabilities (sport classes S1-S10, male and female). The swimmers in these sport classes may have impaired muscle power, limb deficiency, leg length difference, short stature, hypertonia, ataxia, athetosis and impaired passive range of movement (IPC, 2017). The absence of body segments or the presence of movement restrictions (Figueiredo et al., 2014; Osborough et al., 2015) and great implications for drag production (Oh et al., 2013) seem to cause a high degree of heterogeneity in the vast majority of SWD classes (sport classes S1-S10). In these sport 
classes, generation of propulsion is more accentuated by only one upper limb, with a high SR and small SL values at higher speeds (Osborough, Payton, \& Daly, 2009).

Complementarily, there was no heterogeneity for the SL in sport classes S2 to S6 (for the meta-analysis). Sport class S2 presents a reduced possibility of increasing SL because it includes swimmers with: (i) tetraplegia or polio below the C6 vertebra; (ii) very severe quadriplegia (lesion in the encephalon); and (iii) severe skeletal muscle deterioration (Sampedro et al., 2016). On the other hand, sport class S6 seems to be more consolidated in its Paralympic classification because it does not show heterogeneity for SL for male swimmers in the $100 \mathrm{~m}$. Sport class S6 is characterised by swimmers with complete paraplegia or polio between $\mathrm{T} 9$ and L1; diplegia or moderate hemiplegia; double elbow amputation or elbow/knee amputation; dysmelia; or achondroplasia (Dummer, 1999; Sampedro et al., 2016).

The SR is similar between sport classes S7-S10 according to the results of the metaanalysis (sport classes with lower physical disabilities) for male swimmers in the $100 \mathrm{~m}$. However, there are also similarities between sport classes S2-S6 for the meta-analysis (sport classes with the greatest physical handicaps) in the $100 \mathrm{~m}$. Female swimmers showed no heterogeneity in a broader range for meta-analysis (sport classes S3-S8 and S9-S10) in the $100 \mathrm{~m}$. Differences in SS are reported with increases in SL at a low SR among swimmers with conventional anatomy (Craig \& Pendergast, 1979; Craig, Skehan, Pawelczyk, \& Boomer, 1985). However, if SL is short, the swimmer will have to increase SR to swim faster (Figueiredo et al., 2014), as occurs in short events (Ferreira et al., 2012). There is a relationship between SL and SS, as swimmers present great functional ability (Pelayo et al., 1999) and men have a longer SL than women, but without differences in SR (Daly et al., 2003; Daly, Malone, Vanlandewijck, \& Steadward, 1999b). In addition, the results suggest that participants swim faster early in the competition with increased SR, followed by increased SL in the second part, without impairing a further increase in SR at the end of a $100 \mathrm{~m}$ competition (Daly et al., 2003).

Regarding sport classes that involve visual impairment, despite the high degree of heterogeneity found in the meta-analysis for the SS of male swimmers of sport classes $\mathrm{S} 12-\mathrm{S} 13$ in the $100 \mathrm{~m}$, two publications showed that there is similarity between S12-S13 sport class swimmers in the $100 \mathrm{~m}$ (Daly et al., 2009; Malone et al., 2001), and also in males in the $50 \mathrm{~m}$ (Malone et al., 2001). Similarities were found for SL within sport class $\mathrm{S} 11$ in the $100 \mathrm{~m}$ for males, and within S13 in the $100 \mathrm{~m}$ for females (meta-analysis results). The SL values grew from sport classes with lower visual acuity to those with higher visual acuity for males and females. There was no heterogeneity for SR values between sport classes S12 and S13 in the $100 \mathrm{~m}$ for males and females (meta-analysis results). In this context, the parameter that seems to be most important to explain higher speeds in sport classes S12 and S13 compared to S11 is SL.

Of the three classifications (S11, S12 and S13) for visually impaired athletes, the lower number refers to greater visual impairment '(International Blind Sports Federation, 2012)'. Swimmers in sport class S11 have very low visual acuity or do not perceive light and are required to wear sunglasses (IPC, 2017). Differently, swimmers from sport classes S12 and S13 have greater visual acuity, which allows them to get important information on a series of procedures necessary to be able to swim with more speed, positioning and technique refinement (Malone et al., 2001). 
A study was found on SS, SL and SR outcomes in swimmers with intellectual disabilities (Daly et al., 2006). For this case, there is no subgroup comparison, because there is only one sport class (meta-analysis data). When analysing the results of the same publication, there was a certain similarity for SS $(1.68 \pm 0.09 \mathrm{~m} / \mathrm{s})$, slightly lower results for SL $(1.91 \pm 0.03 \mathrm{~m})$ and higher results for SR $(53.25 \pm 3.00 \mathrm{~Hz})$ compared to swimmers in sport class S13 (SS $1.67 \pm 0.09 \mathrm{~m} / \mathrm{s}$; SL $2.04 \pm 0.05 \mathrm{~m}$; and SR $49.66 \pm 2.09 \mathrm{~Hz}$ ) (Daly et al., 2006).

Studies with less possibility of comparison due to differences in protocols, several impairment groups and a case study were also found. This is the case for studies on IdC, IVV and tethered swimming force. The catch-up model was adopted by swimmers with amputation (sport classes S8 and S9, $\mathrm{n}=13$ ) for short distances and in progressive velocity protocols (Osborough et al., 2010) and those in sport class S9 in $50 \mathrm{~m}$ competitions but with only one swimmer with an amputation $(\mathrm{n}=1)$ (Willig et al., 2012). Swimmers classified from S3 to S10 (several impairment groups) demonstrated a different IdC in the $100 \mathrm{~m}$ front crawl (Satkunskiene et al., 2005) with: (i) a catch-up model for those with the lowest functional level, competitive level, SS and minor SL; (ii) a low-degree overlapping model for those with higher SS and SL; and (iii) a higher degree of overlap for those with higher SR and level of competitiveness based on the world record (Van Tilborgh, Daly, Vervaecke, \& Persyn, 1984).

While the IdC and IdCadapt reflect the time interval between the propulsive phases of the arm strokes (Chollet et al., 2000; Osborough et al., 2010; Willig et al., 2012) and therefore the ability to coordinate these propulsive actions (Schnitzler, Seifert, Ernwein, \& Chollet, 2008), IVV is considered a parameter of swimming efficiency. It can provide information on propulsive and resistive actions (Barbosa et al., 2013; Vilas-Boas, Barbosa, \& Fernandes, 2010). At least for swimmers with upper-limb amputations or malformations, that is, swimmers in sport class S9, SR is responsible for the magnitude of IVV in short tests, but it deals with only one case $(n=1)$ (Figueiredo et al., 2014; Willig et al., 2012). This IVV behaviour reflects direct and indirect results of the mechanics and high segment velocities in the most propulsive phases of the swimming cycle, with less drag in the less propulsive phases determining the highest SS and lowest IVV (Barbosa et al., 2010).

The parameters of SR and SL are important for improving performance (Craig et al., 1985; Daly et al., 1999a). Swimmers must explore different motor solutions, facilitating the discovery and adoption of optimum coordination standards in individualised competitive races (Seifert et al., 2011). The coordination of arms is modified with speed increases or decreased length of test, depending on the level of performance (Millet, Chollet, Chalies, \& Chatard, 2002; Schnitzler et al., 2008), respecting the anthropometric characteristics (Toussaint \& Beek, 1992). In that sense, at least in a short race, perhaps the key to gain more speed is in increases with the smallest possible decrease in SL. However, each coach and swimmer must evaluate the main parameters to be optimised on a case by case basis, according to the potentialities and peculiarities of every swimmer (Barbosa et al., 2010; Figueiredo et al., 2014), e.g., swimmers should get an increase also in their SL if they understand that these are important within the association of SL and SR.

No study was found on PE; there is a gap in science about how SWD express their mechanical power to overcome drag in relation to the total mechanic power generated. Perhaps the great morphological variability, different characteristics in performance 
during swimming (Burkett et al., 2018) and possibly different methods used to assess PE (Figueiredo, Toussaint, Vilas-Boas, \& Fernandes, 2013; Zamparo, 2006; Zamparo, Vicentini, Scattolini, Rigamonti, \& Bonifazi, 2012) make these evaluations complex, yet still relevant for a comprehensive analysis of this biomechanical parameter.

This review found only two studies about the force obtained in tethered swimming tests. For those with disability in one of the upper limbs, the force applied during the test (30 s tests) and SR decrease throughout the test (Lee et al., 2014). This result is consistent with the use of high SR to increase SS in unilateral upper-limb swimmers (Osborough et al., 2009). Swimming performance is related to the ability to use the available energy to apply propulsive force during swimming (Morouço, Sacadura, Amaro, \& Matos, 2010). In this sense, the $30 \mathrm{~s}$ tethered swimming test has been used to estimate anaerobic capacity (Morouço et al., 2011), representing measurements of propulsive forces in swimming (Keskinen, 1997; Psycharakis, Paradisis, \& Zacharogiannis, 2011). Although tethered swimming is different from freestyle swimming (Yeater, Martin, White, \& Gilson, 1981) and is not efficient in measuring technique and the difference in active drag force suffered by swimmers, muscle and physiological activity is quite similar between free and tethered front crawl, and it is a valid and reliable method with moderate association to swimming performance (swim time and velocity) (Boilens, Anemans, \& Clarys, 1988; Nagle Zera et al., 2018). The average maximum force correlates to maximum velocity and to SL (Keskinen, Tilli, \& Komi, 1989). The SR is similar between both conditions for a similar test duration (Morouço, Marinho, Keskinen, Badillo, \& Marques, 2014).

The main swimming distances found in this study are short (50 and $100 \mathrm{~m})$. Studies with longer distances, e.g., 200 and $400 \mathrm{~m}$, are, in part, for short protocols or tests developed at swimming pace (Osborough et al., 2015) or at maximal effort to obtain critical swimming velocity (Garatachea et al., 2006). Maybe there is more scientific production on short events because these are also commonly the fastest and with a higher number of eligible sport classes (IPC, 2017). Thus, hypotheses two, three and four of the present study cannot be fully accepted by an insufficient number of studies with a greater number of sport classes.

Regarding the limitations of the present study, quality analysis of observational studies required subjective adaptations in the quality assessment scale for cohort studies, even if these adaptations are common and suggested for this instrument (Costa et al., 2015; Downs \& Black, 1998; Hebert-Losier et al., 2014; Mills et al., 2013). In addition, a possible bias is encountered due to the time interval between metaanalysis studies, i.e., 1992-2013 (Daly et al., 2003, 1999a; Malone et al., 2001; Pelayo et al., 1999; Willig et al., 2012; Zrnzevic, 2016), as performance increases over the time. In this sense, it is necessary to consider that the performance of swimmers over the years has improved.

\section{Practical implications}

The present study provided an opportunity to make recommendations for SWD. The analysis of SR and SL indicates that swimmers can benefit more from SR vs SL when there are disabilities in the upper limbs, e.g. amputations and malformations. The increased SR can also be applied to visually impaired swimmers. However, long-term changes are more dependent on SL (Daly et al., 2003, 2006). Most SWD should seek 
a low IVV, high IdC (overlap model) and training that increases the resultant force applied during swimming. In this way, the training should be designed to increase SR, but one should consider the influence of this increase on a case by case basis, with direct implications in SS, IdC, IVV and the resultant force applied during swimming.

\section{Conclusion}

The current meta-analysis found that SS, SL and SR increase the lower the functional impairment found. Among these parameters, SR should be valued more than SL to achieve high SS in swimmers with disabilities in the upper limbs, e.g. amputations and malformations. However, long-term changes in SS are more dependent on SL than SR. The higher SS in sport classes S12 and S13 compared to S11 in male and female swimmers (in $100 \mathrm{~m}$ front crawlers) seem to be explained by higher SL. However, these results suggest the need for more in-depth studies regarding the classification of the functional system in order to better and more fairly distinguish these swimmers.

\section{Disclosure statement}

No potential conflict of interest was reported by the authors.

\section{ORCID}

Wellington G. Feitosa (D) http://orcid.org/0000-0002-8523-3940

Ricardo de Assis Correia (D) http://orcid.org/0000-0002-2137-2423

Tiago M. Barbosa (D) http://orcid.org/0000-0001-7071-2116

Flávio A. de Souza Castro (iD) http://orcid.org/0000-0003-0848-8226

\section{References}

Almena, A., Pérez-Tejero, T., Coterón, J., \& Veiga, S. (2015). Race analysis in Spanish swimmers with physical disabilities at 100 freestyle event: Influence of funtional classification. Revista de Entrenamiento Deportivo, 29.

Barbosa, T. M., Bragada, J. A., Reis, V. M., Marinho, D. A., Carvalho, C., \& Silva, A. J. (2010). Energetics and biomechanics as determining factors of swimming performance: Updating the state of the art. Journal of Science and Medicine in Sport, 13, 262-269. doi:10.1016/j. jsams.2009.01.003

Barbosa, T. M., Keskinen, K. L., Fernandes, R., Colaco, P., Lima, A. B., \& Vilas-Boas, J. P. (2005). Energy cost and intracyclic variation of the velocity of the centre of mass in butterfly stroke. European Journal of Applied Physiology, 93, 519-523. doi:10.1007/s00421-004-1251-x

Barbosa, T. M., Lima, F., Portela, A., Novais, D., Machado, L., Colaço, P., ... Vilas-Boas, J. P. (2006). Relationships between energy cost, swimming velocity and speed fluctuation in competitive swimming strokes. Portuguese Journal of Sport Sciences, 6, 192-194. doi:10.1055/ s-2005-837450

Barbosa, T. M., Morouço, P. G., Jesus, S., Feitosa, W. G., Costa, M. J., Marinho, D. A., ... Garrido, N. D. (2013). The interaction between intra-cyclic variation of the velocity and mean swimming velocity in young competitive swimmers. International Journal of Sports Medicine, 34, 123-130. doi:10.1055/s-0032-1312582 
Boilens, E., Anemans, L., \& Clarys, J. P. (1988). Peripheral EMG comparison between fully tethered and free front crawl swimming. In B. Ungerechts, K. Wilke, \& K. Reischle (Eds.), Swimming Science V (Vol. 3, pp. 173-181). Champaign: Human Kinetics Publishers.

Borenstein, M., Hedges, L. V., Higgins, J. P., \& Rothstein, H. R. (2009). Introduction to metaanalysis (pp. 69-72). UK: John Wiley \& Sons, Ltd.

Borenstein, M., Hedges, L. V., Higgins, J. P., \& Rothstein, H. R. (2010). A basic introduction to fixed-effect and random-effects models for meta-analysis. Research Synthesis Methods, 1, 97-111. doi:10.1002/jrsm.12

Burkett, B., Payton, C., Van de Vliet, P., Jarvis, H., Daly, D., Mehrkuehler, C., ... Hogarth, L. (2018). Performance characteristics of para swimmers: How effective is the swimming classification system? Physical Medicine and Rehabilitation Clinics of North America, 29, 333-346. doi:10.1016/j.pmr.2018.01.011

Chatard, J. C., Lavoie, J. M., Ottoz, H., Randaxhe, P., Cazorla, G., \& Lacour, J. R. (1992). Physiological aspects of swimming performance for persons with disabilities. Medicine \& Science in Sports \& Exercise, 24, 1276-1282. doi:10.1249/00005768-199211000-00013

Chollet, D., Chalies, S., \& Chatard, J. C. (2000). A new index of coordination for the crawl: Description and usefulness. International Journal of Sports Medicine, 21, 54-59. doi:10.1055/ s-2000-8855

Costa, M. J., Balasekaran, G., Vilas-Boas, J. P., \& Barbosa, T. M. (2015). Physiological adaptations to training in competitive swimming: A systematic review. Journal of Human Kinetics, 49, 179-194. doi:10.1515/hukin-2015-0120

Craig, A. B., Jr., \& Pendergast, D. R. (1979). Relationships of stroke rate, distance per stroke, and velocity in competitive swimming. Medicine and Science in Sports, 11, 278-283. doi:10.1249/ 00005768-197901130-00011

Craig, A. B., Jr., Skehan, P. L., Pawelczyk, J. A., \& Boomer, W. L. (1985). Velocity, stroke rate, and distance per stroke during elite swimming competition. Medicine \& Science in Sports \& Exercise, 17, 625-634. doi:10.1249/00005768-198512000-00001

Daly, D., Djobova, S. K., Malone, L. A., Vanlandewijck, Y., \& Steadward, R. D. (2003). Swimming speed patterns and stroking variables in the paralympic $100 \mathrm{~m}$ freestyle. Adapted Physical Activity Quarterly, 20, 260-278. doi:10.1123/apaq.20.3.260

Daly, D., Einarsson, I., Van de Vliet, P., \& Vanlandewijck, Y. (2006). Freestyle race success in swimmers with intellectual disability. Portuguese Journal of Sports Sciences, 6, 294-296.

Daly, D., Malone, L. A., Burkett, B., Gabrys, T., \& Satkunskiene, D. (2009). Is sight the main deterrent to race performance in visually impaired competitive swimmers?. Physical Education and Sport, 7, 1-15.

Daly, D., Malone, L. A., Vanlandewijck, Y., \& Steadward, R. D. (1999a). Analysis of the men's $100 M$ freestyle at the 1996 Atlanta Paralympic Games. Biomechanics and medicine in swimming VIII. Proceedings of the VIII International Symposium on Biomechanics and Medicine in Swimming, University of Jyvaskyla, Finland, Department of Biology of Physical Activity.

Daly, D., Malone, L. A., Vanlandewijck, Y., \& Steadward, R. D. (1999b, June 30-July 6 1999). Comparison of men's and women's $100 \mathrm{~m}$ freestyle performances at the 1996 Paralympic Games. Scientific proceedings: ISBS '99: XVII International Symposium on Biomechanics in Sports, Edith Cowan University, Perth, Western Australia, Perth School of Biomedical and Sports Science, Edith Cowan University.

Dingley, A. A., Pyne, D., \& Burkett, B. (2014). Dry-land bilateral hand-force production and swimming performance in paralympic swimmers. International Journal of Sports Medicine, 35 (11), e4. doi:10.1055/s-0034-1384590

Downs, S. H., \& Black, N. (1998). The feasibility of creating a checklist for the assessment of the methodological quality both of randomised and non-randomised studies of health care interventions. Journal of Epidemiology and Community Health, 52, 377-384. doi:10.1136/ jech.52.6.377

Dummer, G. M. (1999). Classification of swimmers with psysical disabilities. Adapted Physical Activity Quarterly, 16, 216-218. doi:10.1123/apaq.16.3.216 
Ferreira, M. I., Barbosa, T. M., Costa, M. J., Neiva, H. P., \& Marinho, D. A. (2016). Energetics, biomechanics, and performance in masters' swimmers: A systematic review. The Journal of Strength \& Conditioning Research, 30, 2069-2081. doi:10.1519/JSC.0000000000001279

Ferreira, M. I., Silva, A. J., Oliveira, D. R., Garrido, N. D., Barbosa, T. M., Marinho, D. A., \& Reis, V. M. (2012). Analysis of the determinant kinematical parameters for performance in the 200-m freestyle swimming event. Motriz. Journal of Physical Education, 18, 366-377. doi:10.1590/S1980-65742012000200017

Figueiredo, P., Seifert, L., Vilas-Boas, J. P., \& Fernandes, R. J. (2012). Individual profiles of spatio-temporal coordination in high intensity swimming. Human Movement Science, 31, 1200-1212. doi:10.1016/j.humov.2012.01.006

Figueiredo, P., Toussaint, H. M., Vilas-Boas, J. P., \& Fernandes, R. J. (2013). Relation between efficiency and energy cost with coordination in aquatic locomotion. European Journal of Applied Physiology, 113, 651-659. doi:10.1007/s00421-012-2468-8

Figueiredo, P., Willig, R., Alves, F., Vilas-Boas, J. P., \& Fernandes, R. J. (2014). Biophysical characterization of a swimmer with a unilateral arm amputation: A case study. International Journal of Sports Physiology and Performance, 9, 1050-1053. doi:10.1123/ijspp.2013-0438

Garatachea, N., Abadia, O., Garcia-Isla, F. J., Sarasa, F. J., Bresciani, G., Gonzalez-Gallego, J., \& De Paz, J. A. (2006). Determination and validity of critical swimming velocity in elite physically disabled swimmers. Disability and Rehabilitation, 28, 1551-1556. doi:10.1080/ 09638280600646318

Hay, J. G., \& Guimarães, A. C. S. (1983). A quantitative look at swimming biomechanics. Swimming Technique, 20, 11-17.

Hebert-Losier, K., Supej, M., \& Holmberg, H. C. (2014). Biomechanical factors influencing the performance of elite Alpine ski racers. Sports Medicine, 44, 519-533. doi:10.1007/s40279-013-0132-z

International Blind Sports Federation: Classification rules and procedures, 2016 C.F.R., Cluj Napoca, Romania/IBSA (2012).

International Paralympic Committee [IPC]. Explanatory guide to Paralympic classification in Paralympic summer sports. (2015). Germany: International Paralympic Committee.

International Paralympic Committee [IPC]. (2017). World Para Swimming: classification rules and regulations. Bonn, Germany: International Paralympic Committee.

Keskinen, K. L., \& Komi, P. V. (1993). Intracycle variation in force, velocity and power as a measure of technique performance during front crawl swimming. In S. Bouisset, S. Métral, \& H. Monod (Eds.), XIVth ISB congress of biomechanics (pp. 676-667). France: International Society of Biomechanics.

Keskinen, K. L. (1997). Evaluation of technique performances in freestyle swimming. Kinesiology, 2, 30-38.

Keskinen, K. L., Tilli, L. J., \& Komi, P. V. (1989). Maximum velocity swimming: Interrelationships of stroking characteristics, force production and anthropometric variables. Scandinavian Journal of Sports Sciences, 11, 87-92.

Lee, C. J., Sanders, R. H., \& Payton, C. J. (2014). Changes in force production and stroke parameters of trained able-bodied and unilateral arm-amputee female swimmers during a $30 \mathrm{~s}$ tethered front-crawl swim. Journal of Sports Sciences, 32, 1704-1711. doi:10.1080/ 02640414.2014 .915420

Malone, L. A., Sanders, R. H., Schiltz, J. H., \& Steadward, R. D. (2001). Effects of visual impairment on stroke parameters in Paralympic swimmers. Medicine \& Science in Sports \& Exercise, 33, 2098-2103. doi:10.1097/00005768-200112000-00019

Martin, R. B., Yeater, R. A., \& White, M. K. (1981). A simple analytical model for the crawl stroke. Journal of Biomechanics, 14, 539-548. doi:10.1016/0021-9290(81)90003-8

Millet, G. P., Chollet, D., Chalies, S., \& Chatard, J. C. (2002). Coordination in front crawl in elite triathletes and elite swimmers. International Journal of Sports Medicine, 23, 99-104. doi:10.1055/s-2002-20126

Mills, K., Hunt, M. A., \& Ferber, R. (2013). Biomechanical deviations during level walking associated with knee osteoarthritis: A systematic review and meta-analysis. Arthritis Care \& Research, 65, 1643-1665. doi:10.1002/acr.22015 
Morouço, P. G., Keskinen, K. L., Vilas-Boas, J. P., \& Fernandes, R. J. (2011). Relationship between tethered forces and the four swimming techniques performance. Journal of Applied Biomechanics, 27, 161-169. doi:10.1123/jab.27.2.161

Morouço, P. G., Marinho, D. A., Keskinen, K. L., Badillo, J. J., \& Marques, M. C. (2014). Tethered swimming can be used to evaluate force contribution for short-distance swimming performance. The Journal of Strength \& Conditioning Research, 28, 3093-3099. doi:10.1519/ JSC.0000000000000509

Morouço, P. G., Sacadura, J., Amaro, N., \& Matos, R. (2010). Evaluation of age group swimmers: A in field proposal. The Open Sports Sciences Journal, 3, 38-40. doi:10.2174/ 1875399X01003010038

Nagle Zera, J., Nagle, E. F., Nagai, T., Lovalekar, M., Abt, J. P., \& Lephart, S. M. (2018). Tethered swimming test: Reliability and the association to swimming performance and land-based anaerobic performance. The Journal of Strength \& Conditioning Research. doi:10.1519/ JSC.0000000000002501

Oh, Y. T., Burkett, B., Osborough, C. D., Formosa, D., \& Payton, C. (2013). London 2012 paralympic swimming: Passive drag and the classification system. British Journal of Sports Medicine, 47, 838-843. doi:10.1136/bjsports-2013-092192

Osborough, C. D., Daly, D., \& Payton, C. (2015). Effect of swim speed on leg-to-arm coordination in unilateral arm amputee front crawl swimmers. Journal of Sports Sciences, 33, 1523-1531. doi:10.1080/02640414.2014.996181

Osborough, C. D., Payton, C. J., \& Daly, D. (2009). Relationships between the front crawl stroke parameters of competitive unilateral arm amputee swimmers, with selected anthropometric characteristics. Journal of Applied Biomechanics, 25, 304-312. doi:10.1123/jab.25.4.304

Osborough, C. D., Payton, C. J., \& Daly, D. (2010). Influence of swimming speed on inter-arm coordination in competitive unilateral arm amputee front crawl swimmers. Human Movement Science, 29, 921-931. doi:10.1016/j.humov.2010.05.009

Pelayo, P., Sidney, M., Moretto, P., Wille, F., \& Chollet, D. (1999). Stroking parameters in top level swimmers with a disability. Medicine \& Science in Sports \& Exercise, 31, 1839-1843. doi:10.1097/00005768-199912000-00022

Psycharakis, S. G., Paradisis, G. P., \& Zacharogiannis, E. (2011). Assessment of accuracy, reliability and force measurement errors for a tethered swimming apparatus. International Journal of Performance Analysis in Sport, 11, 411-416. doi:10.1080/24748668.2011.11868560

Sampedro, S. S., Flores, A. A., \& Tejero, J. P. (2016). Performance evolution in paralympic swimmers with physical disabilities: From Beijing 2008 to London 2012. Educación Física Y Deportes, 124, 41-48. doi:10.5672/apunts.2014-0983.es.(2016/2).124.05

Satkunskiene, D., Schega, L., Kunze, K., Birzinyte, K., \& Daly, D. (2005). Coordination in arm movements during crawl stroke in elite swimmers with a loco-motor disability. Human Movement Science, 24, 54-65. doi:10.1016/j.humov.2005.04.001

Schnitzler, C., Seifert, L., Ernwein, V., \& Chollet, D. (2008). Arm coordination adaptations assessment in swimming. [Comparative Study]. International Journal of Sports Medicine, 29, 480-486. doi:10.1055/s-2007-989235

Seidel, W., Klarowicz, A., Rejman, M., \& Szczepan, S. (2016). An evaluation of the usefulness of stroke index values in the swimming training of people with disabilities. Polish Journal of Sport and Tourism, 23, 140-144. doi:10.1515/pjst-2016-0018

Seifert, L., Leblanc, H., Herault, R., Komar, J., Button, C., \& Chollet, D. (2011). Inter-individual variability in the upper-lower limb breaststroke coordination. Human Movement Science, 30, 550-565. doi:10.1016/j.humov.2010.12.003

Sousa, A., Corredeira, R., \& Pereira, A. L. (2013). Paralympic sports in Portugal: From its genesis to present. Portuguese Journal of Sport Sciences, 13, 93-112.

Souto, E. C., Oliveira, L. S., \& Santos Filho, C. S. (2016). The impact of visual impairment on the performance of national and international 50-meter freestyle swimmers. Revista Brasileira De Ciências Da Saúde, 20, 15-20. doi:10.4034/RBCS.2016.20.01.03

Toussaint, H. M., \& Beek, P. J. (1992). Biomechanics of competitive front crawl swimming. Sports Medicine, 13, 8-24. doi:10.2165/00007256-199213010-00002 
Tweedy, S. M., \& Vanlandewijck, Y. C. (2011). International Paralympic Committee position stand-background and scientific principles of classification in Paralympic sport. British Journal of Sports Medicine, 45, 259-269. doi:10.1136/bjsm.2009.065060

Van Tilborgh, L., Daly, D., Vervaecke, H., \& Persyn, U. (1984). The evaluation of some crawl performance determinant factors in women competitive swimmers. In R. H. In, J. Borms, A. Sand, C. Suzzanne, \& M. Hebbelinck (Eds.), Human growth and development (pp. 666-676). New York, NY: Plenum Press.

Viechtbauer, W. (2010). Conducting meta-analyses in R with the metafor package. Journal of Statistical Software, 36. doi:10.18637/jss.v036.i03

Vilas-Boas, J. P., Barbosa, T. M., \& Fernandes, R. J. (2010). Speed fluctuation, swimming economy, performance and training in swimming. In L. Seifert, D. Chollet, \& I. Mujika (Eds.), World book of swimming: From science to performance (pp. 119-134). New York, NY: Nova Science Publishers.

Vrabel, M. (2015). Preferred reporting items for systematic reviews and meta-analyses. Oncology Nursing Forum, 42, 552-554. doi:10.1188/15.ONF.552-554

Wallace, B. C., Schmid, C. H., Lau, J., \& Trikalinos, T. A. (2009). Meta-analyst: Software for meta-analysis of binary, continuous and diagnostic data. BMC Medical Research Methodology, 9, 80. doi:10.1186/1471-2288-9-80

Willig, R. M., Figueiredo, P., Ribeiro, J., Tribuzi, S., Alves, F., Vilas-Boas, J. P., \& Fernandes, R. J. (2012). Biomechanical characterization of the $50 \mathrm{~m}$ freestyle race of an upper limb physically disabled female swimmer. Portuguese Journal of Sport Sciences, 12, 98-109.

Yeater, R. A., Martin, R. B., White, M. K., \& Gilson, K. H. (1981). Tethered swimming forces in the crawl, breast and back strokes and their relationship to competitive performance. Journal of Biomechanics, 14, 527-537. doi:10.1016/0021-9290(81)90002-6

Zamparo, P. (2006). Effects of age and gender on the propelling efficiency of the arm stroke. European Journal of Applied Physiology, 97, 52-58. doi:10.1007/s00421-006-0133-9

Zamparo, P., Pendergast, D. R., Termin, B., \& Minetti, A. E. (2002). How fins affect the economy and efficiency of human swimming. Journal of Experimental Biology, 205, 2665-2676.

Zamparo, P., Vicentini, M., Scattolini, A., Rigamonti, M., \& Bonifazi, M. (2012). The contribution of underwater kicking efficiency in determining 'turning performance' in front crawl swimming. The Journal of Sports Medicine and Physical Fitness, 52, 457-464.

Zrnzevic, J. (2016). Race analysis of swimming performances at Paralympic Games (Master Thesis). University of Leuven - Faculty Kinesiology and Rehabilitations Sciences, Leuven. 